of laboratory staff as measured by fall in errors, improvement in competency assessment scores, attendance in training programs and compliance with standard precautions, documentation and record keeping). A totally new parameter included in this study was the testing of Covid-19 positive patients for HIV, with a positivity of $2.08 \%$.

Conclusion In conclusion, this study yielded some interesting revelations on various aspects of working in an HIV testing laboratory. It has clearly demonstrated that adversities can be used as opportunities for improvement in work ethics, documentation, service providing skills and practice of universal work precautions.

\section{P337 HIV PARTNER NOTIFICATION AMONG MSM LIVING WITH HIV IN GUANGDONG PROVINCE, CHINA: FINDINGS FROM A CROSS-SECTIONAL STUDY}

X Yan*, Y Xu, W Tang, J Tucker, W Miller. UNC Project-China

10.1136/sextrans-2021-sti.387

Background HIV partner notification is critical in preventing HIV transmission, but not widely used in many low and middle-income countries, including China. We conducted a crosssectional survey to investigate the usage of partner notification (PN) for MSM living with HIV in China, and to identify factors that could influence their PN decisions towards different types of sexual partners.

Methods The study recruited MSM who were diagnosed as HIV positive in the past two years in six cities of Guangdong province, China. All participants were $\geq 18$ years old and informed consented. Descriptive analysis was used to report the partner notification rates among different types of sexual partners, along with the top facilitators and barriers for PN. Logistic regressions were used to examine correlates of PN.

Results Among 5799 MSM with HIV, 1376 clicked the link and 944 completed the survey. Mean age was 29 (SD:7.6) and $674(70.8 \%)$ identified gay. Overall, 65.1\% (300/461), $46.4 \%(194 / 416)$ and $54.5 \%(30 / 55)$ notified at least one of their stable, casual, and female partners, respectively. $26.7 \%$ $(165 / 617)$ of the stable male partners tested as HIV positive, while $6.8 \%(74 / 1091)$ of the casual partners tested positive. Important facilitators included stepwise disclosure to friends and HIV support groups, relational intimacy, and anonymous notification, while important barriers included anticipated HIV stigma and fear of discrimination.

Conclusions This study presents the most updated HIV PN data among MSM and contributes to the knowledge of PN in China by differentiating the partner types. It identifies important factors that could influence stable and casual PN decisions, which offers great implications for designing tailored partner services intervention in further research.

\section{P338 SYNERGISTIC ACTIVITY OF AZITHROMYCIN PLUS CEPHALOSPORIN DUAL THERAPY FOR MULTIDRUG- RESISTANT N. GONORRHOEAE IN INCREASING RESISTANCE ENDEMIC SETTING: A CHINA REGIONAL STUDY}

X Lin* . Dermatology Hospital, Southern Medical University

10.1136/sextrans-2021-sti.388
Background The emergence of multidrug resistance (MDR) in Neisseria gonorrhoeae is concerning, especially the co-occurrence of azithromycin resistance (AZMR) and decreased cephalosporin susceptibility (ECSD). We evaluated the feasibility of azithromycin and cephalosporin dual therapy to provide a treatment solution for MDR N. gonorrhoeae strains in Guangdong, China.

Methods A total of 3,609 N. gonorrhoeae strains were collected, and the minimum inhibitory concentrations (MICs) of various antibiotics were assessed for each strain using the agar dilution method. Among the identified MDR strains, 45 isolates were selected and analyzed by $\mathrm{N}$. gonorrhoeae sequence typing for antimicrobial resistance (NG-STAR), N. gonorrhoeae multi-antigen sequence typing (NG-MAST), multi-locus sequence typing (MLST), and phylogenetic tree. The effectiveness of dual therapy was scored by a combination of the MIC and the fractional inhibitory concentration (FIC) index.

Results Among the selected isolates, a high degree of antibiotics resistance was observed: $11.54 \%$ of strains were resistant to AZM; $9.25 \%$ of strains displayed decreased susceptibility to ceftriaxone (CROD); and $11.84 \%$ were less susceptible to cefixime (CFMD). The proportions of isolates with both AZMR and ECSD were once up to $2.11 \%$ for AZMR/CFMD and $1.79 \%$ for AZMR/CROD. NG-STAR, NG-MAST, and MLST categorized the $45 \mathrm{MDR}$ isolates into 35,

35 , and 23 major genogroups, respectively, which could be divided into different evolutionary branches due to genetic diversity. These isolates could be effectively killed with the coadministration of less than $1 \mathrm{mg} / \mathrm{L} \mathrm{AZM}$ and $0.125 \mathrm{mg} / \mathrm{L}$ ECS, with a synergistic effect of FIC $<0.5$.

Conclusions AZM plus ECS dual therapy remains effective against MDR N. gonorrhoeae.

\section{P339 IMPACT OF A NEW RAPID SPECIALIST SEXUAL HEALTH RESULT SERVICE ON TIME FROM TESTING TO TREATMENT OF CHLAMYDIA TRACHOMATIS}

${ }^{1} \mathrm{~S}$ Cochrane, ${ }^{2} \mathrm{R}$ Gardiner, ${ }^{2} \mathrm{M}$ Clarke, ${ }^{3} \mathrm{M}$ Cox, ${ }^{4} \mathrm{P}$ Muir, ${ }^{4} \mathrm{~J}$ Steer, ${ }^{4} \mathrm{R}$ Hopes, ${ }^{2} \mathrm{H}$ Wheeler, ${ }^{2,5} \mathrm{~L}$ Harryman, ${ }^{2} \mathrm{M}$ Crofts, ${ }^{2} \mathrm{~S}$ Moses, ${ }^{2,5} \mathrm{P}$ Horner* ${ }^{*}{ }^{1}$ Royal United Hospitals Bath NHS Foundation Trust, Bath, UK; ${ }^{2}$ University Hospitals Bristol and Weston NHS Foundation Trust, Bristol, UK; ${ }^{3}$ University of Cambridge, Cambridge, UK; ${ }^{4}$ South West Regional Laboratory, Public Health England, Bristol, UK; ${ }^{5}$ University of Bristol, Bristol, UK

\subsection{6/sextrans-2021-sti.389}

Background Unity Sexual Health, Bristol UK, introduced a Panther (Hologic Inc) system in November 2018 at point-ofcare. This rapid Neisseria gonorrhoeae (GC) and Chlamydia trachomatis (CT) nucleic acid amplification test (NAAT) provides results to patients within 4-48 working hours compared with 1-2 weeks previously. Patients are now only treated if CT NAAT-positive, symptomatic and unable to wait for results, or a sexual contact of CT/GC within the preceding 2 weeks. We hypothesised the new service would result in more rapid treatment of $\mathrm{CT} / \mathrm{GC}$ infections. We evaluate the effect on time to CT treatment.

Methods All new CT NAAT-positive cases attending over a 2month period, 1 year before and 1 year after the introduction of the new service were evaluated (01/01/2018-28/02/2018 and 01/01/2020-28/02/2020). Dates of NAAT testing and treatment were obtained from electronic patient records. The one-sided Mann-Whitney U-test was used to compare time to treatment before and after service introduction. 
Results 134 CT-positive patients were diagnosed before and 116 were diagnosed after service introduction. Of these, 2 $(1.5 \%)$ and $8(6.9 \%)$ respectively were treated elsewhere and excluded. Average time to treatment decreased from 6.5 to 4.1 days $(\mathrm{p}=0.09)$. When we excluded patients treated as contacts of CT (before NAAT result was available) the time to treatment decreased from 8.7 to 5.1 days $(p<0.001)$. The reduction in time to treatment decreased significantly more in women; 11.3 to 6.1 days $(\mathrm{p}<0.001)$ than men; 6.8 to 4.7 days $(\mathrm{p}=0.27)$.

Conclusions Introduction of a rapid STI service significantly reduced time from testing to treatment of CT. Availability of microscopy for symptomatic men allows immediate treatment of urethritis, a common presentation of CT in men. This probably explains why men were treated earlier than women. Earlier treatment is expected to reduce both asymptomatic CT transmission, and risk of CT complications, particularly in women.

\section{P340 POTENTIAL EFFECTS OF ANTIBACTERIAL MOUTHWASH ON NEISSERIA GONORRHOEAE TRANSMISSION AMONG MEN WHO HAVE SEX WITH MEN: A MATHEMATICAL MODELLING STUDY}

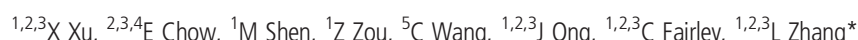
${ }^{1} X i$ 'an Jiaotong University, Xi'an City, China; ${ }^{2}$ Melbourne Sexual Health Centre, Melbourne, Australia; ${ }^{3}$ Monash University, Melbourne, Australia; ${ }^{4}$ The University of Melbourne, Melbourne, Australia; ${ }^{5}$ Zhengzhou University, Zhengzhou City, China

\subsection{6/sextrans-2021-sti.390}

Background Three randomised controlled trials have either reported that mouthwash may increase the susceptibility of the oropharynx to Neisseria gonorrhoeae or potentially decrease its transmissibility. We modelled these potential impacts on gonorrhoea incidence.

Methods We calibrated a susceptible-infected-susceptible compartmental model to examine the effectiveness of antibacterial mouthwash on the transmission of Neisseria gonorrhoeae in men who have sex with men (MSM). Four scenarios include: (1) mouthwash had no effect; (2) mouthwash increased the susceptibility of the oropharynx to Neisseria gonorrhoeae; (3) mouthwash reduced the transmissibility of Neisseria gonorrhoeae from the oropharynx; (4) we combined the effect of mouthwash from scenarios 2 and 3 .

Results Under scenario 1, the overall incidence of gonorrhoea was 44 (95\% CI: 37-50)/100 person-years (PY). Site-specific incidence/100 PY at the oropharynx, anorectum and urethra were 26 (22-31), $9(8-11)$ and 8 (5-12). Under scenario 2, with between $20-80 \%$ mouthwash coverage in the MSM population, the incidence increased at all three anatomical sites by between $7.4 \% \quad(5.9-60.8 \%)$ and $136.6 \%$ (108.1-177.5\%). Under scenario 3, with the same coverage, the incidence decreased at all anatomical sites by between $11.6 \%$ (10.2$13.5 \%$ ) and $99.8 \%$ (99.2-100\%). Under scenario 4, changes in the incidence depended on the efficacy of mouthwash on the transmissibility and susceptibility with both leading to large increases of nearly $130 \%$ or large declines of almost $100 \%$.

Conclusions The effect of mouthwash on gonorrhoea incidence is largely predictable depending on whether it increases the susceptibility to or reduces the transmissibility of Neisseria gonorrhoeae, highlighting an urgent need for further empirical investigation.

\section{P341 PREDICTING THE DIAGNOSIS OF HIV AND SEXUALLY TRANSMITTED INFECTIONS AMONG MEN WHO HAVE SEX WITH MEN USING MACHINE LEARNING APPROACHES}

${ }^{1,2} \mathrm{~N}$ Medland, ${ }^{1,2,3} \mathrm{C}$ Fairley, ${ }^{4} \mathrm{~J} \mathrm{Wu},{ }^{4} \mathrm{X} W \mathrm{Wu}, 1,2,4 \mathrm{E}$ Chow, ${ }^{1,2,3} \mathrm{XXu},{ }^{2} \mathrm{Z} \mathrm{Ge},{ }^{5} \mathrm{X}$ Zhuang $1,2,3$ L Zhang*. ${ }^{2}$ Melbourne Sexual Health Centre, Melbourne, Australia; ${ }^{4}$ Monash University, Melbourne, Australia; 'Xi'an Jiaotong University, Xi'an, China; ${ }^{3}$ The University of Melbourne, Melbourne, Australia; ${ }^{5}$ Nantong University, Nantong City, China

\subsection{6/sextrans-2021-sti.391}

Background We aimed to develop machine learning models and evaluate their performance in predicting HIV and sexually transmitted infections (STIs) diagnosis based on a cohort of Australian men who have sex with men (MSM).

Methods We collected clinical records of 21,273 Australian MSM during 2011-2017. We compared accuracies for predicting HIV and STIs (syphilis, gonorrhoea, chlamydia) diagnosis using four machine learning approaches against a multivariable logistic regression (MLR) model.

Results Machine learning approaches consistently outperformed MLR. Gradient boosting machine (GBM) achieved the highest area under the receiver operator characteristic curve for HIV (76.3\%) and STIs (syphilis, 85.8\%; gonorrhoea, 75.5\%; chlamydia, 68.0\%), followed by extreme gradient boosting (71.1\%, 82.2\%, 70.3\%, 66.4\%), random forest $(72.0 \%$, $81.9 \%, 67.2 \%, 64.3 \%)$, deep learning $(75.8 \%, 81.0 \%, 67.5 \%$, $65.4 \%)$ and MLR (69.8\%, 80.1\%, 67.2\%, 63.2\%). GBM models demonstrated the ten greatest predictors collectively explained $62.7-73.6 \%$ of variations in predicting HIV/STIs. STIs symptoms, past syphilis infection, age, time living in Australia, frequency of condom use with casual male sexual partners during receptive anal sex and the number of casual male sexual partners in the past 12 months were most commonly identified predictors.

Conclusions Machine learning approaches are advantageous over multivariable logistic regression models in predicting HIV/STIs diagnosis.

\section{P342 THE IMPACT OF COVID-19 ON THE SEXUAL HEALTH OF YOUTH IN THE NETHERLANDS}

H De Graaf, F Joemmanbaks*, J Heijne, S Meijer, J Polet. Rutgers, Utrecht, The Netherlands

\subsection{6/sextrans-2021-sti.392}

Background Coronavirus disease (COVID-19) changed people's life drastically, due to restrictions to reduce transmission such as social distancing and the limited number of social contacts. The objective is to gain insight in the impact of the COVID19 pandemic on the sexual health of youth in the Netherlands.

Methods We conducted two cross sectional surveys targeting Dutch youth aged 16-20 year old during the pandemic. Recruitment occurred via social media and a youth sexual health website (sense.info). Both studies included a questionnaire about dating, relationships, sexual- and help seeking behavior and mental health. We identified 4 different time periods: (i) 6 months before the pandemic (pre pandemic), (ii) first lockdown, (iii) between lockdowns, and (iv) second lockdown.

Results The samples consisted of 5218 and 4091 participants. The study showed that less singles had sex during the first $(40 \%)$ and second (52\%) lockdown period compared to pre 